\title{
Malignant Lacrimal Duct Neoplasm
}

National Cancer Institute

\section{Source}

National Cancer Institute. Malignant Lacrimal Duct Neoplasm. NCI Thesaurus. Code C3567.

A primary or metastatic malignant neoplasm affecting the lacrimal duct. 